\title{
LOCALLY INVARIANT TOPOLOGICAL GROUPS AND SEMIDIRECT PRODUCTS
}

\author{
R. W. BAGLEY AND J. S. YANG ${ }^{1}$
}

\begin{abstract}
We consider topological groups which have arbitrarily small invariant neighborhoods of the identity and those topological groups which admit continuous monomorphisms into such groups. We establish conditions under which the two corresponding classes of groups coincide. We apply these results to semidirect products. Since we do not assume local compactness in general, we use the symbol "[Sn]" rather than "[SIN]" for the class of groups with small invariant neighborhoods and the symbol "[In]" for those embeddable in Sn groups. We denote by " $[\mathrm{N}]$ " those groups $G$ with the property: If $\left\{x_{\alpha}\right\}_{\alpha \in D}$ is a net in $G$ which converges to the identity and $\left\{y_{\alpha}\right\}_{\alpha \in D}$ is any net such that $\left\{y_{\alpha} x_{\alpha} y_{\alpha}^{-1}\right\}$ converges, then this net also converges to the identity. We also define a class of topological groups we term $S(U)$ groups. The following are corollaries of our general results: (1) If $G$ is locally compact, $G / G_{0}$ is compact and $G_{0}$ is an $N$ groups, then $G$ is an Sn group. (2) If $H$ is a locally connected compact group, $G$ is an $\mathrm{Sn}$ group, and if the semidirect product $H$ (5) $G$ is an $S(U)$ group, then $H$ (s) $G$ is an Sn group. (3) If $H$ (S $K$ is an $\mathrm{Sn}$ group for every compact group $K$, then every open subgroup of $H$ is of finite index.
\end{abstract}

In this paper we consider the following four classes of topological groups:

[Sn] Those groups which have arbitrarily small neighborhoods of the identity which are invariant under all inner automorphisms. Unless explicitly stated we do not assume local compactness.

[In] Those groups which admit a continuous monomorphism into an Sn group.

[N] Those groups with the property: If $\left\{x_{\alpha}\right\}_{\alpha \in D}$ is a net which converges to the identity and $\left\{y_{\alpha}\right\}_{\alpha \in D}$ is a net such that $\left\{y_{\alpha} x_{\alpha} y_{\alpha}^{-1}\right\}$ converges, then this net also converges to the identity.

$[S(U)]$ Those groups with the property: There exists a neighborhood $U$ of the identity $e$ with the property that for every neighborhood $W$ of $e$, there is a neighborhood $V$ of $e$ such that $g V g^{-1} \subset W \cup(G-U)$ for every $g \in G$.

We shall call a topological group an $A$ group if it belongs to the class [ $A$ ].

E. T. Ordman and S. A. Morris [3] showed that $[\mathrm{In}] \subset[\mathrm{N}]$ and asked if $[\mathrm{N}] \subset[\mathrm{In}]$. There are several situations where [In] and [Sn] are equal. In [3] Ordman and Morris proved that, for locally compact groups $G$ such that $G / G_{0}$ is compact, [Sn] $=[\mathrm{In}]$.

Received by the editors February 22, 1982 and, in revised form, February 22, 1984.

1980 Mathematics Subject Classification. Primary 22A05; Secondary 20E22.

Key words and phrases. Invariant neighborhoods of the identity, nets, semidirect products of topological groups, locally compact groups, compact groups.

${ }^{1}$ The second author wishes to acknowledge the support of a scholar and productive research grant from the University of South Carolina, and to express his appreciation to the University of Miami for the hospitality extended to him during his visit in the fall of 1981. 
We use Proposition 2 below to show that for such groups $G$, if $G_{0}$ is an $\mathrm{N}$ group, then $G$ is an Sn group (Proposition 4). We also relate $S(U)$ groups to the other three classes of groups, and obtain related results for semidirect products.

Throughout this paper, local compactness is not assumed unless otherwise specified. We denote the topological semidirect product obtained by a group $H$ acting on a group $K$ by $H \Subset K$; and the automorphism of $K$ induced by $h \in H$ will be denoted by $\tau_{h}, \tau$ denoting the homomorphism of $H$ into the automorphism group of $K$. The normal subgroups of the product will always be written on the right.

We use the fact that $G$ is an Sn group if and only if, for each net $\left\{x_{\alpha}\right\}_{\alpha \in D}$ in $G$ which converges to the identity and any net $\left\{y_{\alpha}\right\}_{\alpha \in D}$, there is a subnet of $\left\{y_{\alpha} x_{\alpha} y_{\alpha}^{-1}\right\}$ which converges to the identity.

Proposition 1. If $G$ is an $\mathrm{N}$ groups and $G / K$ is an $\mathrm{Sn}$ group, where $K$ is a compact normal subgroup, then $G$ is an $\mathrm{Sn}$ group.

Proof. Let $\left\{x_{\alpha}\right\}$ be a net in $G$ which converges to the identity and let $\left\{y_{\alpha}\right\}$ be any net in $G$. We show that there is a subnet of $\left\{y_{\alpha} x_{\alpha} y_{\alpha}^{-1}\right\}$ which converges to the identity. The net $\left\{y_{\alpha} x_{\alpha} y_{\alpha}^{-1} K\right\}$ converges to the identity in $G / K$ since this group is an Sn group. Thus, for each neighborhood $V$ of the identity of $G$, there exists $\alpha$ such that $y_{\alpha} x_{\alpha} y_{\alpha}^{-1} \in V K$. Thus $y_{\alpha} x_{\alpha} y_{\alpha}^{-1}=v_{\alpha} k_{\alpha}$ for some $v_{\alpha} \in V$ and $k_{\alpha} \in K$. The set $\left\{(\alpha, V): y_{\alpha} x_{\alpha} y_{\alpha}^{-1} \in V K\right\}$ is a directed set with ordering $\left(\alpha_{1}, V_{1}\right) \geqslant\left(\alpha_{2}, V_{2}\right)$ if $\alpha_{1} \geqslant \alpha_{2}$ and $V_{1} \subset V_{2}$. This directed set is the domain for a net which is a subnet of $\left\{y_{\alpha} x_{\alpha} y_{\alpha}^{-1}\right\}$ and that subnet, by virtue of compactness of $K$ and the fact that $v_{\alpha} \rightarrow e$, contains a subnet which converges to some $k \in K$. Since $G$ is an $\mathrm{N}$ group, $k=e$; and this final subnet is the one desired.

We will refer to the following corollary later.

CoRollary. If $H$ is an $\mathrm{Sn}$ group, $K$ is compact and $H$ (s $K$ is an $\mathrm{N}$ group, then $H$ (S $K$ is an $\mathrm{Sn}$ group.

Proposition 2. If $G$ is an $\mathrm{N}$ group and $K$ is a compact normal subgroup, then $G / K$ is an $\mathrm{N}$ group.

The proof of this proposition is essentially the same as that of Proposition 1.

Proposition 3. If $\boldsymbol{H}$ is a locally connected group which acts equicontinuously on an Sn group $G$ and the corresponding semidirect product $H \Subset G$ is an $S(U)$ group, then $H$ (s) $G$ is an $\mathrm{Sn}$ group.

Proof. We can assume that the neighborhood $U$ satisying the hypothesis on $H$ (s) $G$ is a rectangular neighborhood $N \times M$. If $V$ and $W$ are open neighborhoods of the identities of $H$ and $G$, respectively, such that $V \times W \subset N \times M$, we choose neighborhoods $V_{1}$ and $W_{1}$ such that $\tau_{h}\left(W_{1}\right) \subset W$ for all $h \in H, W_{1}$ is invariant under the inner automorphisms of $G, V_{1}$ is connected and

$$
(h, g)\left(V_{1} \times W_{1}\right)(h, g)^{-1} \subset(V \times W) \cup(H \times G-\bar{N} \times \bar{M})
$$

for all $(h, g) \in H \times G$. Now let $(h, g)$ be any element of $H \times G$ and $w \in W_{1}$. The sets $A=\left\{h v h^{-1}: v \in V_{1}\right\}$ and $B=\left\{\tau_{h^{-1}}\left(\tau_{v}(g) w g^{-1}\right): v \in V_{1}\right\}$ are connected, so 
$A \times B$ is connected. By taking $v=e$ we see that $A \cap V \neq \varnothing$ and $B \cap W \neq \varnothing$, thus $(A \times B) \cap(V \times W) \neq \varnothing$. The connectedness of $A \times B$ implies that $A \times B \subset V \times$ $W$ and completes the proof.

COROLLARY 1. If $H$ is a locally connected $S(U)$ group, then $H$ is an $\mathrm{Sn}$ group.

COROLlaRY 2. If $H$ is a locally connected compact group, $G$ is an Sn group, and if the semidirect product $H$ (s) $G$ is an $S(U)$ group, then $H$ (s) $G$ is an $\mathrm{Sn}$ group.

The above corollaries are immediate consequences of Proposition 3. The proof of the following proposition is straightforward and thus will be omitted.

Proposition 4. If $H$ is a locally compact $\mathrm{N}$ group, then $H$ is an $S(U)$ group for any neighborhood $U$ of the identity such that $\bar{U}$ is compact.

COROLlaRY 1. If $H$ is a locally connected compact group, $G$ is a locally compact $\mathrm{Sn}$ group, and if the semidirect product $H$ (S) $G$ is an $\mathrm{N}$ group, then $H$ (5) $G$ is an $\mathrm{Sn}$ group.

Proof. By Proposition 4, $H$ @ $G$ is an $S(U)$ group. Since $H$ is compact, it acts equicontinuously on $G$; the conclusion follows from Proposition 3.

Another consequence of Proposition 4 is the following, which was proved in [3].

COROLlaRY 2. If $H$ is a locally connected, locally compact $\mathrm{N}$ group, then $H$ is an $\mathrm{Sn}$ group.

Proof. This corollary follows from Proposition 4, and Corollary 1 of Proposition 3.

The following is an apparent generalization of Theorem $A$ of [3].

Proposition 5. If $G$ is a locally compact group, $G / G_{0}$ is compact and $G_{0}$ is an $\mathrm{N}$ group, then $G$ is an $\mathrm{Sn}$ group.

Proof. Suppose that $G_{0}$ is an $\mathrm{N}$ group. Since $G_{0}$ is locally compact and connected, there is a compact normal subgroup $K$ such that $G_{0} / K$ is a Lie group. Now $G_{0} / K$ is an $\mathrm{N}$ group by Proposition 2 and thus an Sn group by Corollary 2 of Proposition 4. It follows from Proposition 1 that $G_{0}$ is an $\operatorname{Sn}$ group and therefore MAP. Thus $G$ is an $\mathrm{Sn}$ group by [2, Theorem 2.9 and its corollary].

We have two corollaries of the above results.

COROLlARY 1. If $G$ is an In group and $G / K$ is compactly generated, where $K$ is a compact normal subgroup, then $G$ is an $\mathrm{Sn}$ group.

Proof. By Proposition 2 of [3], $G / K$ is an In group and hence an Sn group by Theorem 8 (1) of [4]. The conclusion follows from Proposition 1 above, noting again that any In group is an $\mathrm{N}$ group.

COROLlARY 2. If $G$ is a locally compact $\mathrm{N}$ group and $G / K$ is locally connected, where $K$ is a compact normal subgroup, then $G$ is an $\mathrm{Sn}$ group.

Proof. By Proposition 3, $G / K$ is an $\mathrm{N}$ group and hence an Sn group by Corollary 2 of Proposition 4. The conclusion now follows from Proposition 1. 
The following two propositions with the corollary to Proposition 2 give a partial answer to the question: What can be said about a group $H$ if every semidirect product $H$ (s) $K$ is an $\mathrm{Sn}$ group where $K$ is any compact group?

Proposition 6. If $G$ is an $\mathrm{Sn}$ group which is the semidirect product obtained by a compact group $C$ acting on a connected group $H$, then any semidirect product $G \Subset K$, where $K$ is compact, is an $\mathrm{Sn}$ group.

Proof. We let $\tau$ be the homomorphism from $G$ into the automorphism group of $K$ which determines $G \Subset K$. We express the elements of $G$ as pairs $(c, h)$, where $c \in C$ and $h \in H$, and note that $\tau(e \times H)$ is contained in the inner automorphism group of $K$ by Theorem 2 of [1]. Now, if $\left\{\left(x_{\alpha}, u_{\alpha}, y_{\alpha}\right)\right\}$ is a net in $C \times H \times K$ which converges to the identity and $\left\{\left(c_{\alpha}, h_{\alpha}, k_{\alpha}\right)\right\}$ is any net, then the first two coordinate nets of $\left\{\left(c_{\alpha}, h_{\alpha}, k_{\alpha}\right)\left(s_{\alpha}, u_{\alpha}, y_{\alpha}\right)\left(c_{\alpha}, h_{\alpha}, k_{\alpha}\right)^{-1}\right\}$ converge to the respective identities of $C$ and $H$ since $G$ is an $\mathrm{Sn}$ group. We show that the third coordinate converges to the identity of $K$. Since $C$ and $K$ are compact and it is sufficient to show that a subnet of the original net in $G \circledast K$ converges to the identity, we can assume that $\left\{c_{\alpha}\right\}$ and $\left\{k_{\alpha}\right\}$ converge. The third coordinate in question can be expressed as follows: $\tau_{\left(e, h_{\alpha}^{-1}\right)} \tau_{\left(c_{\alpha}^{-1}, e\right)}\left(\tau_{\left(x_{\alpha}, u_{\alpha}\right)}\left(k_{\alpha}\right) y_{\alpha} k_{\alpha}^{-1}\right)$. Since $\tau_{\left(e, h_{\alpha}^{-1}\right)}$ is an inner automorphism of $K$, $K$ is compact and $\tau_{\left(c_{\alpha}^{-1}, e\right)}\left(\tau_{\left(x_{\alpha}, u_{\alpha}\right)}\left(k_{\alpha}\right) y_{\alpha} k_{\alpha}^{-1}\right)$ converges to the identity of $K$, we have the desired conclusion.

COROLlaRY. If $G$ is a locally compact $\mathrm{Sn}$ group such that $G / G_{0}$ is compact and $K$ is any compact group, then any semidirect product $G$ (S $K$ is an $\mathrm{Sn}$ group.

Proof. $G$ has the structure $C$ (s) $V$, where $C$ is compact and $V$ is a vector group.

Proposition 7. If $G$ @ $K$ is an $\mathrm{Sn}$ group for every compact group $K$, then every open subgroup of $G$ has finite index, and if $G$ is locally connected, then $G / G_{0}$ is finite.

Proof. We first show that, if $G$ is an infinite discrete group, then there is a compact group $K$ and a semidirect product $G$ (\$) which is not an $\mathrm{Sn}$ group. We let $K=Z_{2}^{G}$ with the product topology where $Z_{2}$ is the two element discrete group. We define the automorphism $\tau_{g}$ for $g \in G$ as follows: For $x \in G$, the $x$-coordinate of $\tau_{g}(k)$ is the $g x$-coordinate of $k$. It is clear that the mapping, $\phi: G \times K \rightarrow K$ defined by $\phi(g, k)=\tau_{g}(k)$ is continuous since $G$ is discrete. To see that $G \circledast K$ is not an Sn group let $\left\{g_{n}\right\}$ be a sequence of distinct elements of $G$ and define a sequence $\left\{x_{n}\right\}$ in $K$ as follows: The $g_{n}^{-1}$-coordinate of $x_{n}$ is 1 and all other coordinates are 0 . Obviously $x_{n} \rightarrow 0 \in k$. However, the sequence $\left\{\left(g_{n}, 0\right)\left(e, x_{n}\right)\left(g_{n}^{-1}, 0\right)\right\}$ converges to $(e, k)$ where the $e$-coordinate of $k$ is 1 .

Now suppose that $G$ has an open subgroup $H$ of infinite index so that $G / H$ is discrete. From the above example we have a compact group $K$ and a semidirect product $G / H$ (S $K$ which is not an $\mathrm{Sn}$ group. From this we define a semidirect product $G$ (s) in an obvious way and note that $G$ (s $K$ is not an Sn group. This completes the proof since, in the locally connected case, $G_{0}$ is open.

We note that the example constructed in the proof of Proposition 7 is not an $\mathrm{N}$ group and hence not an In group. The following is a simple example of an MAP 
group which is not an Sn group. The construction does not require proof of the existence of representations or almost periodic functions.

EXAMPLE. Let $K=\Pi_{n \in Z} K_{n}$ with the product topology, where $K_{n}=Z_{2}$ for each $n$. Let $D=\sum_{n \in Z} D_{n}$, the direct sum, with the discrete topology, where $D_{n}=Z_{3}$ for each $n$. We define the semidirect product $K \odot D$ by the formula $(k, x)(h, y)=(k+$ $h, h(x)+y)$, where the sums are coordinatewise and $h(x)$ has $n$th coordinate the $n$th coordinate of $x$ if the $n$th coordinate of $h$ is 0 and the $n$th coordinate of $-x$ if the $n$th coordinate of $h$ is 1 . To see that $K \odot D$ is not an Sn group let $\left\{k_{n}\right\}$ be a sequence in $K$ such that the $n$th coordinate of $k_{n}$ is 1 and all other coordinates are 0 . Take $\left\{x_{n}\right\}$ in $D$ such that the $n$th coordinate of $x_{n}$ is 1 and all other coordinates are 0 . Now $\left\{\left(k_{n}, 0\right)\right\} \rightarrow(0,0)$ but $\left(0, x_{n}\right)\left(k_{n}, 0\right)\left(0,-x_{n}\right)=\left(k_{n},-x_{n}\right)$ which does not converge since $\left\{x_{n}\right\}$ is not eventually constant. To see that $K \circledast D$ is MAP we let $C=\prod_{n \in Z} D_{n}$ with the product topology and $D_{n}$ as above. We define $K \odot C$ with the same group operation as in $K \odot D$. Since injection of $K \odot D$ into $K \oplus C$ is a continuous monomorphism and $K \odot C$ is compact, the desired conclusion follows.

Proposition 8. If $H$ is an $\mathrm{Sn}$ group and $V$ is a vector group such that $H$ (S $V$ is an $\mathrm{N}$ group, then $H$ \& $V$ is an $\mathrm{Sn}$ group.

Proof. Suppose $\left(x_{\alpha}, y_{\alpha}\right) \rightarrow(e, 0)$ in $H$ (S) Let $\left\{\left(h_{\alpha}, v_{\alpha}\right)\right\}$ be any net. Then

$$
\left(h_{\alpha}, v_{\alpha}\right)\left(x_{\alpha}, y_{\alpha}\right)\left(h_{\alpha}, v_{\alpha}\right)^{-1}=\left(h_{\alpha} v_{\alpha} h_{\alpha}^{-1}, \tau_{h_{\alpha}}^{-1}\left(\tau_{x_{\alpha}}\left(v_{\alpha}\right)+y_{\alpha}-v_{\alpha}\right)\right) \text {. }
$$

Since $H$ is an $\mathrm{Sn}$ group the first coordinate net converges to $e$ in $H$. Suppose $z_{\alpha}=\tau_{h_{\alpha}}^{-1}\left(\tau_{x_{\alpha}}\left(v_{\alpha}\right)+y_{\alpha}-v_{\alpha}\right)$ does not converge to 0 . Then there is a subnet $\left\{z_{\alpha_{\beta}}\right\}$ outside some neighborhood of 0 . That is, $a_{\beta}=\left\|z_{\alpha_{\beta}}\right\|$ is bounded away from 0 . Thus the net $\left\{x_{\beta}, y_{\beta} / a_{\beta}\right\}$ converges to $(e, 0)$, writing " $x_{\beta}=x_{\alpha_{\beta}}$ ", etc. Now

$$
\left(h_{\beta}, v_{\beta} / a_{\beta}\right)\left(x_{\beta}, y_{\beta} / a_{\beta}\right)\left(h_{\beta}, v_{\beta} / a_{\beta}\right)^{-1}=\left(h_{\beta} x_{\beta} h_{\beta}^{-1},\left(1 / a_{\beta}\right) \tau_{h_{\beta}}^{-1}\left(\tau_{x_{\beta}}\left(v_{\beta}\right)+y_{\beta}-v_{\beta}\right)\right) \text {. }
$$

Since $V$ is locally compact and the norm of the second coordinate is equal to 1 for all $\beta$, some subnet converges to $v \neq 0$. The corresponding subnet of the original net of conjugates converges to $(e, v)$, contradicting the hypothesis that $H \Subset V$ is an $\mathrm{N}$ group. This completes the proof.

COROLlaRY. If $K \oplus V$ is an $\mathrm{N}$ group, where $K$ is compact and $V$ is a vector group, then $\tau(K)$, the set of automorphisms on $V$ induced by $K$, is finite.

Proof. Since $K \propto V$ is an Sn group, the conclusion follows from 2.9 of [2].

T. S. Wu suggested the proof of the following (cf. [4, Theorem 8(1)]):

Proposition 9. If $G$ is a compactly generated $\mathrm{N}$ group, then $G$ is an $\mathrm{Sn}$ group.

Proof. Suppose $G$ is generated by a compact neighborhood of the identity, $W=K$. Let $W_{0}$ be an open neighborhood of $e$ such that $k W_{0} k^{-1} \subset W$ for each $k \in K$. Take a neighborhood base $\left\{W_{\alpha}\right\}$ of $e$ such that $k W_{\alpha} k^{-1} \subset W_{0}$ for $k \in K$ and all $\alpha$. Suppose no $W_{\alpha}$ satisfies $g W_{\alpha} g^{-1} \subset W$ for $g \in G$. Then, for each $\alpha$, there exist $x_{\alpha} \in W_{\alpha}$ and $k_{1}, \ldots, k_{n_{\alpha}} \in K$ such that $k_{1} \cdots k_{n_{\alpha}} x_{\alpha} k_{n_{\alpha}}^{-1} \cdots k_{1}^{-1} \notin W$. There is 
a largest $j, 1<j<n_{\alpha}$, such that $k_{j} \cdots k_{n_{\alpha}} x_{\alpha} k_{n_{\alpha}}^{-1} \cdots k_{j}^{-1} \notin W_{0}$, but $k_{j} \cdots$ $k_{n_{\alpha}} x_{\alpha} k_{n_{\alpha}}^{-1} \cdots k_{j}^{-1} \in W$. Thus $k_{j} \cdots k_{n_{\alpha}} x_{\alpha} k_{n_{\alpha}}^{-1} \cdots k_{j}^{-1} \in W-W_{0}$. Since $W-W_{0}$ is compact and $\left\{x_{\alpha}\right\}$ converges to $e$, this contradicts the fact that $G$ is an N group, and the proof is completed.

Proposition 9 gives us a shorter alternate proof of Proposition 5 as follows: Since $G_{0}$ is a compactly generated $\mathrm{N}$ group, $G_{0}$ is $\operatorname{SIN}$ by Proposition 9 , and hence is MAP. Thus $G$ is MAP [2, Corollary 2.10], and consequently $G$ is an $S_{n}$ group by Theorem 2.9 of [2].

We close with two remarks whose proofs are simple.

REMARK 1. An $\mathrm{N}$ group is an $\mathrm{Sn}$ group if and only if every net of the form $\left\{y_{\alpha} x_{\alpha} y_{\alpha}^{-1}\right\}$, where $\left\{x_{\alpha}\right\}$ converges to the identity, has a convergent subnet.

It follows immediately from this remark that an $\mathrm{N}$ group which has a compact invariant neighborhood of the identity is an Sn group.

REMARK 2. If $H$ is an $\mathrm{N}$ group, $K$ is compact and $H$ (s $K$ is a semidirect product such that $\tau(H)$ is contained in the inner automorphism group of $K$, then $H$ (s) is an $\mathrm{N}$ group.

\section{REFERENCES}

1. R. W. Bagley and K. K. Lau, Semidirect products of topological groups with equal uniformities, Proc. Amer. Math. Soc. 29 (1971), 179-182.

2. S. Grosser and M. Moskowitz, Compactness conditions in topological groups, J. Reine Angew. Math. 246 (1971), 1-40.

3. E. T. Ordman and S. A. Morris, Almost locally invariant topological groups, J. London Math. Soc. (2) 9 (1974), 30-34.

4. R. T. Ramsay, Groups with equal uniformities, Canad. J. Math. 21 (1969), 655-659.

Department of Mathematics, University of Miami, Coral Gables, Florida 33124

Department of Mathematics, University of South Carolina, Columbia, South Carolina 29208 